\title{
Single Case Experimental Design: A New Approach for Non-invasive Brain Stimulation Research?
}

\author{
Joshua W. Pate and Alana B. McCambridge* \\ Graduate School of Health, Discipline of Physiotherapy, University of Technology Sydney, Sydney, NSW, Australia
}

Keywords: single case experimental design, non-invasive brain stimulation, repeated transcranial magnetic stimulation, transcranial direct current stimulation, patient-tailored

\section{INTRODUCTION}

Due to the large proportion of people living with chronic neurological impairments, noninvasive brain stimulation (NIBS) was developed as a potential adjuvant to enhance neurological rehabilitation. NIBS techniques such as transcranial direct current stimulation (tDCS) and repetitive transcranial magnetic stimulation (rTMS) have been proposed to temporarily modulate neural excitability in a given direction based on the type of stimulation used. For example, seminal studies in tDCS reported that anodal tDCS over the motor cortex (M1) increased corticomotor excitability, whereas M1 cathodal tDCS decreased corticomotor excitability, for up to an hour

OPEN ACCESS

Edited by:

Wei-Peng Teo,

Nanyang Technological

University, Singapore

Reviewed by:

Onno van der Groen,

Edith Cowan University, Australia

*Correspondence:

Alana B. McCambridge

alana.mccambridge@uts.edu.au

Specialty section:

This article was submitted to

Augmented and Synthetic

Neuroergonomics,

a section of the journal

Frontiers in Neuroergonomics

Received: 09 March 2021

Accepted: 10 May 2021

Published: 31 May 2021

Citation:

Pate JW and McCambridge AB (2021) Single Case Experimental Design: A

New Approach for Non-invasive Brain

Stimulation Research?

Front. Neuroergon. 2:678579.

doi: 10.3389/fnrgo.2021.678579
(Nitsche and Paulus, 2001; Nitsche et al., 2003). The polarity dependent changes in corticomotor excitability were suggested to represent up-regulation or down-regulation of neural plasticity (Nitsche and Paulus, 2001; Nitsche et al., 2003) and depend on several stimulation parameters (Woods et al., 2016). When delivered before or during rehabilitation NIBS may increase the beneficial neuroplastic effects of rehabilitation alone.

Subsequently, the field of NIBS grew rapidly. Many researchers applied tDCS or rTMS to a wide range of clinical populations and explored many variations in experimental parameters. Most commonly, researchers opted to use a randomized controlled trial (RCT) study design, allocating participants to either; real vs. sham stimulation, facilitatory vs. inhibitory stimulation (e.g., anodal vs. cathodal tDCS), stimulation alone vs. stimulation with therapy, or some combination of the above in a multi-arm trial. In addition, NIBS trials have typically used functional and/or neurophysiological outcomes (such as motor learning tasks or measures of corticomotor excitability after M1 stimulation) to determine the after-effects of stimulation, often followed up at multiple time-points to determine the duration of effects. The ease and reported effectiveness of sham stimulation (e.g., fade in-fade out protocol for tDCS, or sham coil rTMS) (Gandiga et al., 2006; Mennemeier et al., 2009) allows robust methodological study designs such as double-blinded cross-over RCTs frequently used in NIBS research.

However, despite the rapid rise of NIBS research over the past 20 years, current evidence has largely not supported translation into clinical practice, with only rTMS as a treatment for drugresistant depression adopted clinically so far (O'Connell et al., 2018; Elsner et al., 2020). One of the major criticisms of NIBS that likely acts as a barrier to translation is the substantial between-subject variability observed in response to stimulation (López-Alonso et al., 2014; Wiethoff et al., 2014; McCambridge et al., 2015). With some causal studies of healthy participants showing that only half of participants respond to NIBS as expected (López-Alonso et al., 2014; Wiethoff et al., 2014). Therefore, when investigating group-level changes in outcomes the results are unlikely to show statistical or clinically meaningful differences and may mask the positive benefits experienced by some participants. Yet, it is not surprising that substantial between-subject variability exists given 
the lack of precision in current NIBS studies that typically adopt a "one-size-fits-all" approach to delivery of the intervention.

In the literature, several determinants of NIBS are known to influence stimulation response, both interventional (e.g., type and location of stimulation) and biological factors (e.g., skull thickness, brain morphology and neurochemistry) (Filmer et al., 2019; Hordacre et al., 2021) that should be taken into consideration when delivering NIBS. Researchers have discussed the need for individualized or tailored stimulation protocols, using known determinants of NIBS to guide stimulation protocols to help address wide-scale variability in responses (Di Pino et al., 2014; McCambridge et al., 2018; Hordacre et al., 2021). For example in tDCS research, individualized stimulation could tailor the stimulation protocol based on clinical characteristics of the patient (e.g., lesion location, structural integrity of neural pathways) (Di Pino et al., 2014) or individually modeled electric fields (Antonenko et al., 2019) or a combination of many factors including methodological (e.g., dosage and biological factors) (Hordacre et al., 2021) (Figure 1). Patienttailored brain stimulation would be a sensible approach, though the feasibility of conducting a large-scale RCT with highly-precise individualized stimulation protocols for each participant would be difficult, particularly in a clinical population. Therefore, in this opinion article, we consider if an alternate methodological approach to investigating the effectiveness of NIBS in lab-based or clinical settings would be of interest to the field.

\section{SINGLE-CASE EXPERIMENTAL DESIGNS}

To our knowledge, a study design that has not yet been employed in NIBS research is a single case experimental design (SCED). SCEDs are clinical trials of an individual (or several individuals each studied as a single case), in contrast to trials of groups of participants (Tate and Perdices, 2019). They have also been called single-system designs, single-subject designs, single-case research designs, single participant designs, interrupted timeseries designs, and $\mathrm{N}$-of- 1 trials. As with many other study designs, the results of SCEDs can be included in systematic reviews (Tanious and Onghena, 2020). To establish a causal relationship between a target behavior and an intervention, SCEDs have four essential elements: (1) the participant is their own control, (2) a priori methods are used, (3) the target behavior is repeatedly measured before, during and after an intervention, and (4) the intervention is systematically manipulated (Tate and Perdices, 2019). Inferences about the intervention are then drawn from the repeated demonstration of the intervention effect on the outcome. Table 1 shows the four classifications of SCED designs: (1) withdrawal/reversal designs, (2) multiple-baselines designs, (3) alternating-treatments designs, and (4) changingcriterion designs. A range of modifications and combinations of these four approaches have been used to date (Shadish and Sullivan, 2011), with some designs better suited to particular type of research questions (see Table 1). One key appeal of a SCED is the ability to individualize an intervention to a given participant, as would be done in many clinical practice settings. For health research, SCEDs are considered Level 1 evidence
(OCEBM Levels of Evidence Working Group, 2011) and the methodological quality of SCEDs can be assessed and guided by the recently developed Single-Case Reporting guideline In BEhavioural interventions (SCRIBE) 2016 checklist (Tate et al., 2016a,b).

\section{EXAMPLE PROTOCOL FOR NON-INVASIVE BRAIN STIMULATION}

\section{Planning}

To further elaborate on SCEDs and the potential utility for NIBS research, we propose an example SCED protocol to answer the research question "Is motor cortex anodal tDCS more effective than sham tDCS at increasing corticomotor excitability in chronic stroke participants?" To answer this question, a withdrawal multiple treatment design is appropriate (see Table 1). In this example, an A-B-C-B-C-A design could be used where $\mathrm{A}=$ baseline or follow-up with no stimulation, $\mathrm{B}$ $=$ sham $\mathrm{tDCS}$, and $\mathrm{C}=$ real $\mathrm{tDCS}$. This design provides three opportunities to examine the experimental effect between $\mathrm{B}$ and C. To establish the absolute effectiveness of B or C compared to baseline, the design would require at least three phase changes involving $\mathrm{A}$ and $\mathrm{C}$, and three involving $\mathrm{A}$ and $\mathrm{B}$. This is a very cumbersome design [e.g., A-B-C-B-C-A-B-A-C-A (Tate and Perdices, 2019)]. One ethical and practical consideration here is whether the design should end on an active intervention phase so that the study ends on a potentially beneficial treatment for the patient. The appropriate duration of each phase is dependent on the "wash-out" period needed after stimulation, estimated to be $1-1.5 \mathrm{~h}$ for anodal tDCS (Nitsche and Paulus, 2001) thereby minimizing possible confounding "alternation effects" (Tate and Perdices, 2019). Within each phase, at least five measurements of the outcome measure should be taken (see Figure 1B for hypothetical data) to account for possible variability (Kratochwill et al., 2013). In this example, 10 TMS-pulses could be delivered every 4-5 s at each measurement to provide a total of five average MEP amplitudes per phase.

Randomization and blinding are important for the internal validity of a SCED. Randomization can be achieved in some SCED classifications by randomizing the phases, or randomizing the onset in a multiple-baseline study. In our A-B-C-B-C-A example, the sequence of phases is not randomized. However, blinding could be achieved by concealing the stimulation type (real, sham) to the participants and the outcome assessors and not revealing the order of phases or having a separate researcher administer the intervention that is not involved in data collection or analysis. For example, sham tDCS protocols set up the electrodes the same as in real stimulation except the current is ramped up to provide sensory stimulation on the scalp and then ramped down again to no stimulation. Some tDCS machines can also be pre-programmed to do the "fade in-fade out" protocol without the experimenter's knowledge or use a separate experimenter deliver the stimulation. In addition, blinding during the analysis could be achieved by not disclosing the phase (baseline, real, sham, follow up) to the statistician analyzing the data until all analyses are complete. 
A

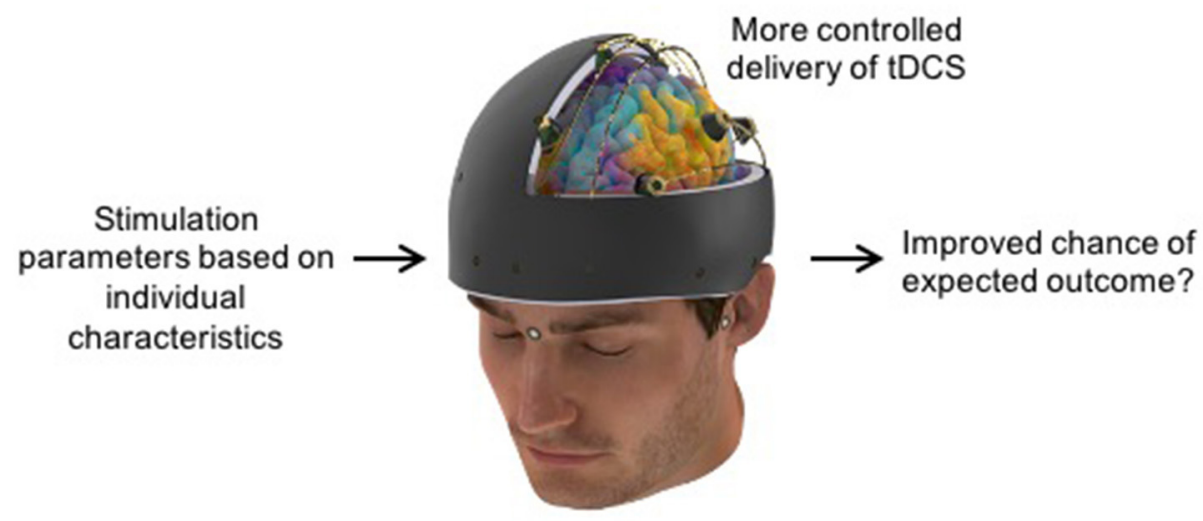

B

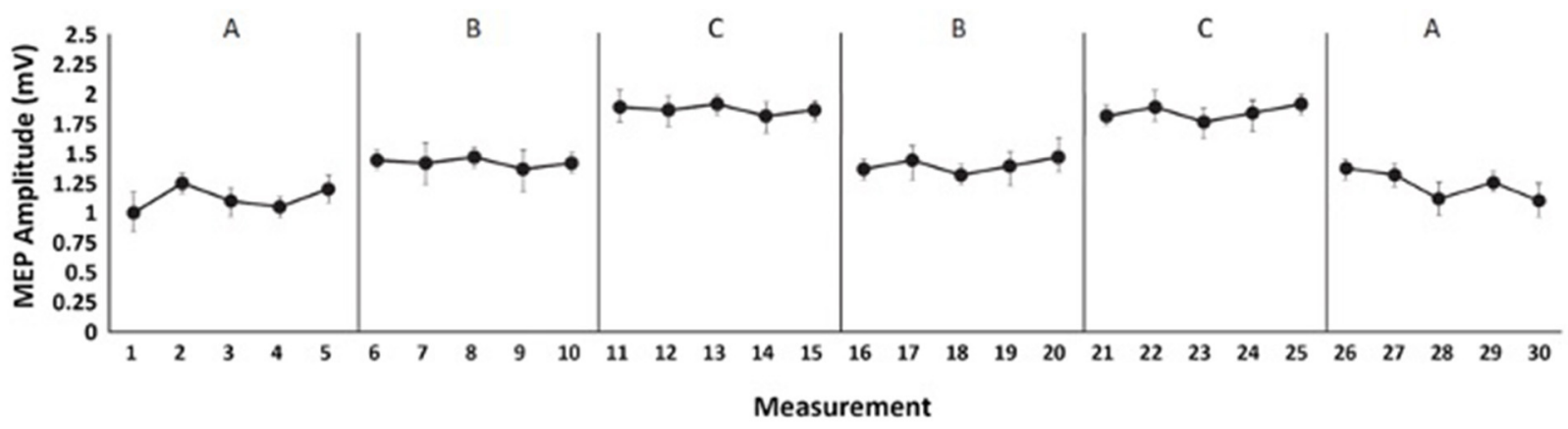

FIGURE 1 | (A) Example of individualized transcranial direct current stimulation (tDCS) that could be tested using SCED methodology. (B) Hypothetical data for an A-B-C-B-C-A design where five measurements are taken in each of the six phases so that three phase changes between $B$ and $C$ can be reported on (e.g., $B \rightarrow C, C$ $\rightarrow \mathrm{B}$, and $\mathrm{B} \rightarrow \mathrm{C}$ again).

\section{Considerations}

In terms of reporting on a SCED study, it is important to thoroughly report the selection criteria, participant characteristics, setting, outcome measures, equipment, intervention details, and the procedural fidelity of the intervention. A thorough description is required for replication and to maximize the external validity of the study, for example, researchers may want to repeat the trial on three or more participants that represent the clinical population (i.e., young and old, male and female, different settings etc.).

Detailed descriptions of all phases of the intervention are required (i.e., baseline, real, sham) in SCED studies. If the study uses patient-tailored brain stimulation then a comprehensive description should be given about how stimulation is individualized (i.e., based on what parameters) so that replication of the intervention application and how it was individualized could be achieved in subsequent trials. Reporting how and when the intervention was delivered as well as the intervention dosage (e.g., current strength, duration of stimulation, electrode size and placement etc.) is of particular importance. Similar detail should be given to describing the sham intervention, for example the current strength and duration of the tDCS fade in-fade out protocol (Ambrus et al., 2012).

\section{Analysis}

To begin planning a SCED data analysis, it is important to first determine if changes in level, trend, and/or variability of the outcome measure are indicative of a treatment effect. For example, if the outcome measure was corticomotor excitability assessed with TMS-induced motor evoked potentials (MEP), then a change in the level of corticomotor excitability (i.e., amplitude of the MEPs) with real NIBS compared to sham and baseline would indicate stimulation was effective. Similarly, if the outcome was a motor learning task you may hypothesize a change in the trend (i.e., rate of improvement) with real NIBS would indicate that the treatment was effective. Visual and statistical analysis supplement each other in SCED studies and need to be utilized in conjunction (Tate and Perdices, 2019), though controversy about analysis procedures exist.

The approach selected for visual and statistical analysis is essential to consider. Kratochwill et al. (2013) includes four steps in visually evaluating the phases of a SCED study which can be summarized as: (1) "Is the data stable at baseline?" (2) "Are there any trends or variability?" (3) "How long until there is an effect?" and (4) "Did the control work?" Various techniques exist to visually analyse data in a systematic and objective manner, including descriptive statistics, level changes, split-middle trend lines, variability and evaluating trends in each 
TABLE 1 | Classification of SCED designs adapted from Chapter 1 of Tate and Perdices (2019).

\begin{tabular}{|c|c|c|c|c|}
\hline Classification & Research question & $\begin{array}{l}\text { NIBS example research } \\
\text { question }\end{array}$ & $\begin{array}{l}\text { When would you choose this } \\
\text { approach? }\end{array}$ & Designs \\
\hline $\begin{array}{l}\text { (1) Withdrawal/reversal } \\
\text { designs }\end{array}$ & $\begin{array}{l}\text { What is the effect of systematically } \\
\text { introducing and withdrawing the } \\
\text { intervention on the target behavior? }\end{array}$ & $\begin{array}{l}\text { Does individualized motor } \\
\text { cortex anodal tDCS } \\
\text { compared to sham tDCS } \\
\text { facilitate corticomotor } \\
\text { excitability? }\end{array}$ & $\begin{array}{l}\text { If intervention effects are to be } \\
\text { evaluated relative to baseline } \\
\text { performance }\end{array}$ & $\begin{array}{l}\text { - } \text { A-B-A-B } \\
\text { - } \text { Multiple-treatment } \\
\text { (e.g., A-B-C-B-C-A) }\end{array}$ \\
\hline $\begin{array}{l}\text { (2) Multiple-baselines } \\
\text { designs }\end{array}$ & $\begin{array}{l}\text { What is the effect of an intervention } \\
\text { when applied simultaneously, but in a } \\
\text { staggered sequence, to different } \\
\text { participants, or alternatively, different } \\
\text { target behaviors or different settings } \\
\text { for the same individual? }\end{array}$ & $\begin{array}{l}\text { Does cognitive training with } \\
\text { tDCS whether delivered at } \\
\text { home or in clinic improve } \\
\text { cognition in people with } \\
\text { multiple sclerosis? }\end{array}$ & $\begin{array}{l}\text { If ( } 1 \text { ) the intervention is likely to } \\
\text { produce permanent changes, or (2) it } \\
\text { is unethical/impractical to return to } \\
\text { baseline conditions }\end{array}$ & $\begin{array}{l}\text { - Across participants } \\
\text { - Across behaviors } \\
\text { - Across settings }\end{array}$ \\
\hline $\begin{array}{l}\text { (3) Alternating } \\
\text {-treatments designs }\end{array}$ & $\begin{array}{l}\text { What is the effectiveness of two or } \\
\text { more interventions (one of which can } \\
\text { be a "no-treatment" condition) in the } \\
\text { same participant? }\end{array}$ & $\begin{array}{l}\text { Is ipsilesional facilitatory } \\
\text { stimulation or contralesional } \\
\text { inhibitory stimulation of the } \\
\text { motor cortex most effective } \\
\text { at increasing paretic arm } \\
\text { function after stroke? }\end{array}$ & $\begin{array}{l}\text { If two or more interventions can be } \\
\text { provided to the same participant in } \\
\text { rapid alternation }\end{array}$ & $\begin{array}{l}\text { - Comparison phase only } \\
\text { - Comparison phase with initial } \\
\text { baseline } \\
\text { - Comparison phase with "best } \\
\text { treatment" phase }\end{array}$ \\
\hline $\begin{array}{l}\text { (4) Changing-criterion } \\
\text { designs }\end{array}$ & $\begin{array}{l}\text { How effective is an intervention at } \\
\text { gradually inducing therapeutic } \\
\text { change in the target behavior? }\end{array}$ & $\begin{array}{l}\text { Is NIBS to the pre-frontal } \\
\text { cortex able to gradually } \\
\text { reduce food cravings in } \\
\text { Bulimia nervosa? }\end{array}$ & $\begin{array}{l}\text { If (1) the intervention is not withdrawn, } \\
\text { (2) the same intervention is applied } \\
\text { across all the subphases, and (3) the } \\
\text { level of target behavior performance } \\
\text { in each intervention subphase can } \\
\text { become the "baseline" against which } \\
\text { performance in the next intervention } \\
\text { subphase is compared. }\end{array}$ & $\begin{array}{l}\text { - Standard } \\
\text { - Range-bound } \\
\text { - Distributed criterion }\end{array}$ \\
\hline
\end{tabular}

phase (Parsonson et al., 1992; Lane and Gast, 2014; Barton et al., 2018). For statistical analysis methods, detailed guides are available (Manolov and Solanas Pérez, 2018) because a range of issues may influence the validity of statistical tests (Parker et al., 2011; Velicer and Molenaar, 2013; Harrington and Velicer, 2015). Statistical analyses are valuable (1) when variability appears large upon visual inspection, (2) when effects of interventions are not yet well-understood, (3) when small but important changes in target behaviors cannot be detected by visual analysis, or (4) to enhance replication studies (Kazdin and Tuma, 1982).

\section{ADVANTAGES AND CHALLENGES}

SCEDs are a useful study design to further explore NIBS, particularly for patient-tailored NIBS protocols. Similar to a RCT, SCEDs are considered Level 1 evidence for health interventions (OCEBM Levels of Evidence Working Group, 2011). One advantage of a SCED over a large-scale RCT is that it is more cost effective, because it requires fewer participants and resources. Not requiring large sample sizes to achieve statistical power may be of particular importance for low-incidence patient populations that often suffer from being under-powered. In addition, because SCEDs use an individual as their own control, outcome measures can be interpreted as absolute values relative to the participant's baseline. Interpretation of absolute values would avoid non-validated normalization procedures used in some neurophysiological research (e.g., pre-post, prepost/post, pre-post/pre+post). Although tailored-interventions can be used in RCTs, SCEDs are also suitable for patienttailored treatments that may involve clinical judgements that are commonplace in clinical practice. In a SCED study, individual modifications to the protocol can be extensively explained for each participant so that replication is possible. Furthermore, exploring patient-tailored NIBS with a SCED design can also inform how a tailored intervention could be further tested in a large-scale RCT.

However, there are some important challenges that should be considered. A current challenge to SCED research is the generalizability of findings, particularly for very heterogenous patient populations (e.g., stroke, dystonia). Replication is therefore required, though guidance on how many participants are needed for acceptable replication is lacking. SCED's can also be burdensome to participants because they require extensive data collection. Another challenge is that SCED's are vulnerable to plausible rival hypotheses that may explain the outcomes such as, maturation, regression to the mean and external factors (Caneiro et al., 2019). Further, there are currently no agreed upon statistical analysis procedures. Criticism of the subjective nature of visual inspection, and hence acceptability of SCEDs as Level 1 evidence by the field, could also be a barrier to translation.

\section{SUMMARY}

In summary, the fundamental aspects of NIBS appear to be suitable for studies using SCED methodology. This approach may be a useful avenue to further investigate inter-individual 
variability and more advanced individualized stimulation protocols. In addition, due to the relative ease and safety of modulating the independent variable in NIBS research, NIBS may also be a candidate field to improve and develop SCED methodology.

\section{REFERENCES}

Ambrus, G. G., Al-Moyed, H., Chaieb, L., Sarp, L., Antal, A., and Paulus, W. (2012). The fade-in - short stimulation-fade out approach to sham tDCS—reliable at $1 \mathrm{~mA}$ for naïve and experienced subjects, but not investigators. Brain Stimul. 5, 499-504. doi: 10.1016/j.brs.2011.12.001

Antonenko, D., Thielscher, A., Saturnino, G. B., Aydin, S., Ittermann, B., Grittner, U., et al. (2019). Towards precise brain stimulation: is electric field simulation related to neuromodulation? Brain Stimul. 12, 1159-1168. doi: 10.1016/j.brs.2019. 03.072

Barton, E. E., Lloyd, B. P., Spriggs, A. D., and Gast, D. L. (2018). "Visual analysis of graphic data," in Single Case Research Methodology: Applications in Special Education and Behavioral Sciences, eds J. R. Ledford and D. L. Gast (New York, NY: Routledge), 179-214.

Caneiro, J. P., Smith, A., Linton, S. J., Moseley, G. L., and O’Sullivan, P. (2019). How does change unfold? an evaluation of the process of change in four people with chronic low back pain and high pain-related fear managed with Cognitive Functional Therapy: a replicated single-case experimental design study. Behav. Res. Ther. 117, 28-39. doi: 10.1016/j.brat.2019.02.007

Di Pino, G., Pellegrino, G., Assenza, G., Capone, F., Ferreri, F., Formica, D., et al. (2014). Modulation of brain plasticity in stroke: a novel model for neurorehabilitation. Nat. Rev. Neurol. 10, 597-608. doi: 10.1038/nrneurol.20 14.162

Elsner, B., Kugler, J., Pohl, M., and Mehrholz, J. (2020). Transcranial direct current stimulation (tDCS) for improving activities of daily living, and physical and cognitive functioning, in people after stroke. Cochrane Database Syst. Rev. 3:CD009645. doi: 10.1002/14651858.CD0096 45.pub4

Filmer, H. L., Ehrhardt, S. E., Bollmann, S., Mattingley, J. B., and Dux, P. E. (2019). Accounting for individual differences in the response to tDCS with baseline levels of neurochemical excitability. Cortex 115, 324-334. doi: 10.1016/j.cortex.2019.02.012

Gandiga, P. C., Hummel, F. C., and Cohen, L. G. (2006). Transcranial DC stimulation (tDCS): a tool for double-blind sham-controlled clinical studies in brain stimulation. Clin. Neurophysiol. 117, 845-850. doi: 10.1016/j.clinph.2005.12.003

Harrington, M., and Velicer, W. F. (2015). Comparing visual and statistical analysis in single-case studies using published studies. Multivar. Behav. Res. 50, 162-183. doi: 10.1080/00273171.2014.9 73989

Hordacre, B., McCambridge, A. B., Ridding, M. C., and Bradnam, L. V. (2021). Can transcranial direct current stimulation enhance poststroke motor recovery? development of a theoretical patienttailored model. Neurology doi: 10.1212/WNL.00000000000 12187. [Epub ahead of print].

Kazdin, A. E., and Tuma, A. H. (1982). Single-Case Research Designs. Oxford: Oxford University Press.

Kratochwill, T. R., Hitchcock, J. H., Horner, R. H., Levin, J. R., Odom, S. L., Rindskopf, D. M., et al. (2013). Single-case intervention research design standards. Remedial Spec. Educ. 34, 26-38. doi: 10.1177/0741932512 452794

Lane, J. D., and Gast, D. L. (2014). Visual analysis in single case experimental design studies: brief review and guidelines. Neuropsychol. Rehabil. 24, 445-463. doi: 10.1080/09602011.2013.8 15636

López-Alonso, V., Cheeran, B., Río-Rodríguez, D., and Fernández-Del-Olmo, M. (2014). Inter-individual variability in response to non-invasive brain

\section{AUTHOR CONTRIBUTIONS}

AM: conception of the idea. AM and JP: manuscript preparation and approval of final paper. Both authors contributed to the article and approved the submitted version.

stimulation paradigms. Brain Stimul. 7, 372-380. doi: 10.1016/j.brs.2014. 02.004

Manolov, R., and Solanas Pérez, A. (2018). Analytical options for single-case experimental designs: review and application to brain impairment. Brain Impair. 19, 18-32. doi: 10.1017/BrImp.2017.17

McCambridge, A. B., Stinear, J. W., and Byblow, W. D. (2015). "I-wave" recruitment determines response to tDCS in the upper limb, but only so far. Brain Stimul. 8, 1124-1129. doi: 10.1016/j.brs.2015.07.027

McCambridge, A. B., Stinear, J. W., and Byblow, W. D. (2018). Revisiting interhemispheric imbalance in chronic stroke: a tDCS study. Clin. Neurophysiol. 129, 42-50. doi: 10.1016/j.clinph.2017.10.016

Mennemeier, M., Triggs, W., Chelette, K., Woods, A., Kimbrell, T., and Dornhoffer, J. (2009). Sham transcranial magnetic stimulation using electrical stimulation of the scalp. Brain Stimul. 2, 168-173. doi: 10.1016/j.brs.2009.02.002

Nitsche, M. A., Nitsche, M. S., Klein, C. C., Tergau, F., Rothwell, J. C., and Paulus, W. (2003). Level of action of cathodal DC polarisation induced inhibition of the human motor cortex. Clin. Neurophysiol. 114, 600-604. doi: 10.1016/S1388-2457(02)0 0412-1

Nitsche, M. A., and Paulus, W. (2001). Sustained excitability elevations induced by transcranial DC motor cortex stimulation in humans. Neurology 57, 1899-1901. doi: 10.1212/WNL.57.10.1899

OCEBM Levels of Evidence Working Group (2011). The Oxford Levels of Evidence 2. Oxford: Centre for Evidence-Based Medicine.

O'Connell, N. E., Marston, L., Spencer, S., DeSouza, L. H., and Wand, B. M. (2018). Non-invasive brain stimulation techniques for chronic pain. Cochrane Database Syst. Rev. 3:CD008208. doi: 10.1002/14651858.CD008 208.pub5

Parker, R. I., Vannest, K. J., and Davis, J. L. (2011). Effect size in single-case research: a review of nine nonoverlap techniques. Behav. Modif. 35, 303-322. doi: 10.1177/01454455113 99147

Parsonson, B. S., Baer, D. M., Kratochwill, T., and Levin, J. (1992). “The visual analysis of data, and current research into the stimuli controlling it," in Single-Case Research Design and Analysis: New Directions for Psychology and Education, eds T. R. Kratochwill and J. R. Levin (Mahwah, NJ: Lawrence Erlbaum Associates, Inc.), 15-40.

Shadish, W. R., and Sullivan, K. J. (2011). Characteristics of single-case designs used to assess intervention effects in 2008. Behav. Res. Methods 43, 971-980. doi: 10.3758/s13428-011 $-0111-y$

Tanious, R., and Onghena, P. (2020). A systematic review of applied single-case research published between 2016 and 2018: study designs, randomization, data aspects, data analysis. Behav. Res. Methods doi: 10.3758/s13428-020-01502-4. [Epub ahead of print].

Tate, R., and Perdices, M. (2019). Single-Case Experimental Designs for Clinical Research and Neurorehabilitation Settings: Planning, Conduct, Analysis, and Reporting. London: Routledge.

Tate, R. L., Perdices, M., Rosenkoetter, U., McDonald, S., Togher, L., Shadish, W., et al. (2016b). The Single-Case Reporting Guideline In BEhavioural Interventions (SCRIBE) 2016: explanation and elaboration. Arch. Sci. Psychol. 4, 10-31. doi: 10.1037/arc00 00027

Tate, R. L., Perdices, M., Rosenkoetter, U., Shadish, W., Vohra, S., Barlow, D. H., et al. (2016a). The Single-Case Reporting Guideline In BEhavioural Interventions (SCRIBE) 2016 statement. Phys. Ther. 96, e1-e10. doi: $10.2522 / \mathrm{ptj} .2016 .96 .7 . e 1$ 
Velicer, W., and Molenaar, P. (2013). "T ime series analysis: research methods in psychology," in Volume 2 of Handbook of Psychology, eds J. Schinka and W. F. Velicer (New York, NY: John Wiley and Sons), 628-660.

Wiethoff, S., Hamada, M., and Rothwell, J. C. (2014). Variability in response to transcranial direct current stimulation of the motor cortex. Brain Stimul. 7, 468-475. doi: 10.1016/j.brs.2014. 02.003

Woods, A. J., Antal, A., Bikson, M., Boggio, P. S., Brunoni, A. R., Celnik, P., et al. (2016). A technical guide to tDCS, and related non-invasive brain stimulation tools. Clin. Neurophysiol. 127, 1031-1048. doi: 10.1016/j.clinph.2015.11.012
Conflict of Interest: The authors declare that the research was conducted in the absence of any commercial or financial relationships that could be construed as a potential conflict of interest.

Copyright $\odot 2021$ Pate and McCambridge. This is an open-access article distributed under the terms of the Creative Commons Attribution License (CC BY). The use, distribution or reproduction in other forums is permitted, provided the original author(s) and the copyright owner(s) are credited and that the original publication in this journal is cited, in accordance with accepted academic practice. No use, distribution or reproduction is permitted which does not comply with these terms. 